\title{
CFRP truss for the CCAT 25 m diameter submillimeter-wave telescope
}

\author{
David P. Woody ${ }^{* a}$, Steve Padin ${ }^{\mathrm{b}}$, Thomas Sebring ${ }^{\mathrm{c}}$ \\ ${ }^{a}$ Owens Valley Radio Observatory, California Institute of Technology, 100 Leighton Lane, Big Pine, \\ CA 93513-0968; ' California Institute of Technology, Pasadena CA 91125; 'Cornell University, \\ Center for Radiophysics and Space Research, Ithaca NY
}

\begin{abstract}
CCAT will be a $25 \mathrm{~m}$ diameter submillimeter-wave telescope that will operate inside a dome located on Cerro Chajnantor in the Atacama Desert. The telescope must have high aperture efficiency at a wavelength of 350 microns and good performance out to a wavelength of 200 microns. A conceptual design for a carbon fiber reinforced plastic (CFRP) truss and primary reflector support truss has been developed. This design yields a telescope with a net $1 / 2$ wave front error of $<10$ microns using a lookup table to adjust the segment actuators to compensate for gravitational deflections. Minor corrections may be required to compensate for the expected $20 \mathrm{C}$ temperature excursions. These can be handled using a coarse lookup table.
\end{abstract}

Keywords: Submillimeter telescope, telescope structures, CFRP truss

\section{INTRODUCTION}

CCAT will be a $25 \mathrm{~m}$ diameter submillimeter-wave telescope that will operate inside a dome located on Cerro Chajnantor in the Atacama Desert ${ }^{1}$. The telescope must have high aperture efficiency at a wavelength of 350 microns with good performance out to 200 microns. The critical requirement is to achieve a net $1 / 2$ wave front error (HWFE) of less than 10 microns, including the primary, secondary and tertiary optics. The top down error budget allocates only 6 microns for the primary surface maintenance and stability not including segment fabrication and distortion errors. Widefield instruments for CCAT require a $3 \mathrm{~m}$ diameter clear optical path through the truss and a primary vertex that is only 3 $m$ in front of the elevation axis. These are severe constraints for the primary support structure ${ }^{2}$.

The CCAT project is investigating two approaches to achieving this challenging specification: a CFRP truss with active control of the surface based on lookup tables; and a steel truss with closed loop control based on segment edge sensor measurements. Both approaches have the same 162 segment surface configuration with each segment mounted on three computer controlled actuators. The segment design is described in Woody et $\mathrm{al}^{3}$. The closed loop control approach employing edge sensors is described in two papers at this converence ${ }^{4,5}$.

The CFRP truss approach, presented in this paper, is to control the segments based on a lookup table to correct for gravitational deformations. In this case, the primary truss must have thermal deformations much smaller than the required HWFE. We have developed a truss design that exploits the high strength to weight ratio and low CTE of CFRP to achieve small gravitational and thermal deformations.

A critical aspect of telescopes utilizing CFRP is the interface between the CFRP and steel structures. The large differences in the CTE of these two materials must be accommodated without introducing added distortions or excess stress. These design issues and concepts are discussed in section 2 . Two primary support structure designs are presented in section 3 while section 4 discusses a design for the truss node and struts. The finite element analysis (FEA) results are discussed in section 5 and this work is summarized in section 6 .

"dwoody@caltech.edu; phone1 760-938-2075x111; fax 1 760-938-2075; http://www.submm.org/

Ground-based and Airborne Telescopes III, edited by Larry M. Stepp, Roberto Gilmozzi, Helen J. Hall Proc. of SPIE Vol. $7733,77332 B$ - C 2010 SPIE - CCC code: $0277-786 X / 10 / \$ 18 \cdot$ doi: $10.1117 / 12.856498$ 


\section{THERMAL HOMOLOGY}

A common problem in large precision structures is coupling major subsystems that have different CTEs or are at different temperatures. This is particularly true for large telescopes. Even if the telescope is fabricated entirely out of the same material, such as structural steel, there can be large temperature differences between the massive mount and yoke structure and the low density primary truss structure. Thermal expansion can produce significant distortions in the structure and degrade the primary surface figure.

One solution is that used on the IRAM 30-m telescope in Spain where the primary truss is insulated and a thermal control system with large fans is used to keep the truss close to the same temperature as the yoke ${ }^{6}$. This works well for operation at $1 \mathrm{~mm}$ but is unlikely to be sufficient for operation at 200 microns.

Submillimeter telescopes often use low CTE CFRP for the primary support structure which then presents the problem how to couple this structure to the steel tipping structure and mount. The SubMillimeter Telescope on mount Graham in Arizona and the SubMillimeter Array on Mauna Kea, Hawaii use a circular ring of blades to connect the CFRP structure to the steel tipping platform ${ }^{7}$. This works well but is not as stiff as a fully bolted connection and it limits the configuration options for large beams and instruments on CCAT.

The ALMA Vertex antenna also employs low CTE CFRP in the primary support structure and uses an Invar mating ring to couple to a temperature controlled steel tipping platform and receiver room. This is very stiff but requires a large Invar ring and a temperature controlled steel tipping platform and as with the circular ring of blades limits the telescope configurations.

The designs presented here make use of thermal homology to construct an interface between the CFRP and steel structures that is distributed throughout the 3-D volume between the two elevation bearings. There are two simple principles that can be used in the design of the coupling between two large structures that will experience uniform but different thermal expansion.

1) A structure fabricated from a single isotropic material remains homologous when subject to a uniform temperature change; that is planes remain plains, circles remain circle, straight lines remain lines and the angles between lines do not change.

2) A strut that is oriented perpendicular to a plane and attached to a point on the plane experiences a very small stress or strain when the attachment point is moved a small distance in the plane. A $1 \mathrm{~m}$ long strut in this configuration experience an elongation of only 0.5 micron when the attachment point shifts $1 \mathrm{~mm}$ in the plane. A corollary is that this also applies to a strut that is nominally perpendicular to a line for small motion of the attachment point along the line.

A structure that experiences uniform isotropic expansion has no preferred reference point and you are free to pick a single reference point. If you pick an idealized reference point in free space when the two structures are at the same temperature and undistorted then you can use the above two principles to design a stiff and expansion independent coupling between them. The logical reference point for a ring of blades or kinematic bi-pod connections is the center of the circle containing the blades or bi-pods. The blades are usually relatively short so the motion of the opposite ends of the struts is not necessarily insignificant but because the struts are all the same length and the ends are in a plane, the motion is a uniform translation perpendicular to the plane.

The application of thermal homology principles is not limited to simple attachments in a plane. They can be applied to 3D attachment geometries as shown in figure 1. This figure shows the interface between a central pillar and five nodes of a space frame truss. For clarity the rest of the space frame truss is not shown. The pillar might be a steel plate structure while the truss is fabricated from CFRP struts. Each of the five truss nodes is connected to four corners on the central pillar providing a very stiff interconnect between the pillar and the space frame. The effect of uniform expansion of the pillar relative to the truss will introduce negligible distortion to the truss as long as the struts connecting the two structures are perpendicular to the line from the attachment point to the chosen reference point shown as a red sphere in figure 1. 
The local distortion beyond simple uniform expansion for a temperature change $(\Delta T)$, depends upon the difference in CTE for the two materials $(\triangle \mathrm{CTE})$, length of the connecting strut $\left(\mathrm{L}_{\mathrm{S}}\right)$ and the distance from the chosen center of expansion to the attachment point $\left(\mathrm{L}_{\mathrm{C}}\right)$;

$$
\delta L_{S}=\frac{1}{2} \frac{\left(\Delta T \Delta C T E L_{C}\right)^{2}}{L_{S}}
$$

Interfacing a CFRP truss to a steel structure with an attachment point that is $5 \mathrm{~m}$ from the center of expansion requires a CFRP strut length greater than $0.5 \mathrm{~m}$ to keep the local distortions under 1 micron for a $20 \mathrm{C}$ temperature change. The $3 \mathrm{D}$ distributed interface for the CCAT telescope described in the following section produces $\sim 2$ micron of RMS surface distortion for a $10 \mathrm{C}$ temperature change for CFRP with $\mathrm{CTE}=0.2 \mathrm{ppm} / \mathrm{C}$.

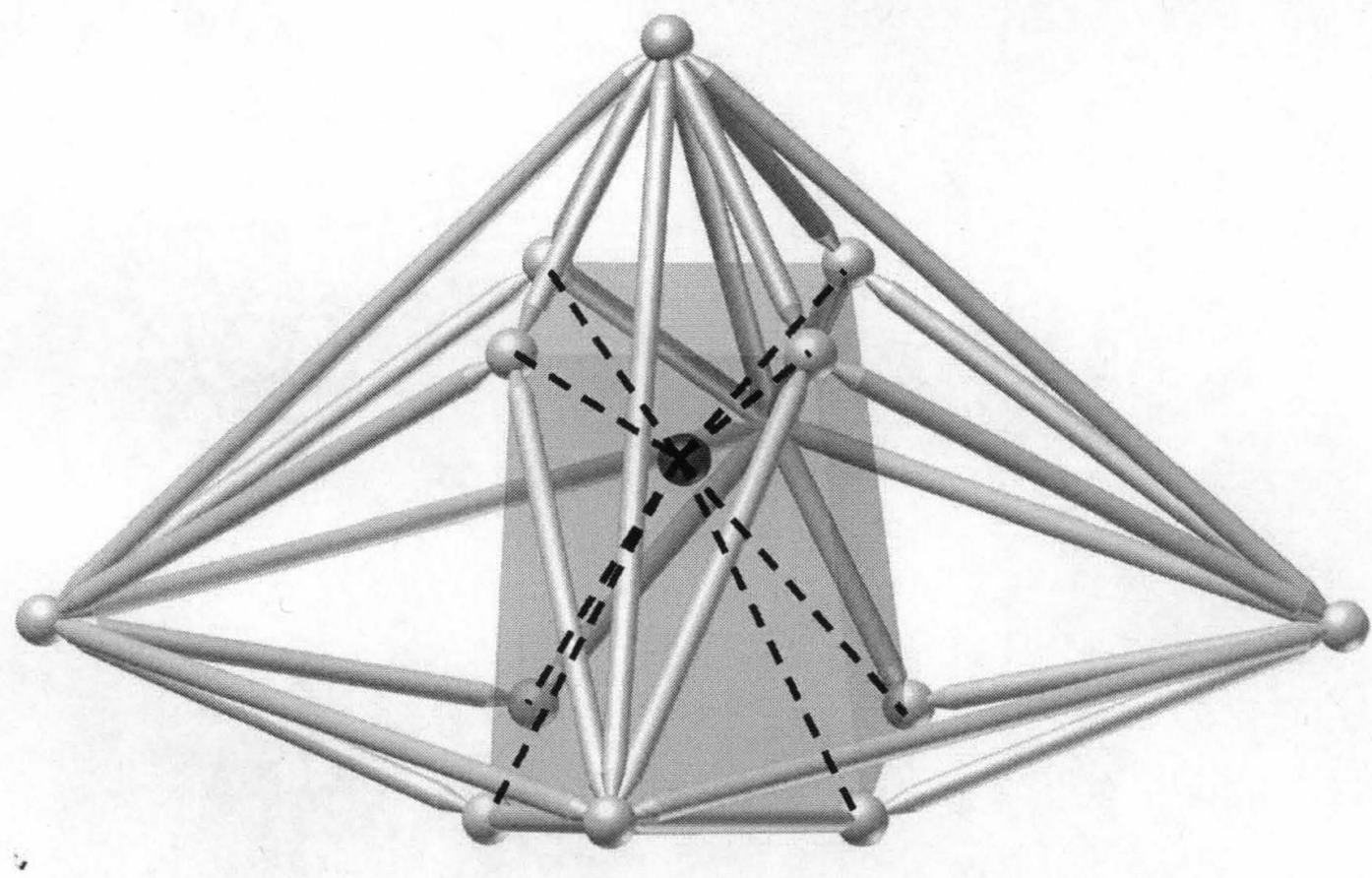

Figure 1. Schematic of a 3D distributed interconnection between a central pillar from one material shown in translucent green and the inner section of a space frame structure shown in gray. The chosen center of expansion is the red (or dark gray) sphere inside the translucent green block. The dashed lines are construction lines extending from the expansion reference point to the eight points where the struts attach to the pillar. The connecting struts are perpendicular these construction lines.

\section{CCAT DESIGN CONCEPT}

The baseline design for the CCAT primary consists of $1622 \mathrm{~m} \times 2 \mathrm{~m}$ compound segments mounted on three actuators. Each segment consists of between 4 and 16 reflector tiles mounted on a CFRP sub-frame ${ }^{3}$. The sub-frame is thermally insulated yielding a very stable structure. Because the tiles are not very large and can be mounted on multiple adjusters several different types of reflector tiles ranging from machined all Aluminum tiles to laminated CFRP-Al honeycombCFRP tiles are likely to meet the segment surface accuracy and stability requirements.

Figure 2 shows one design for the CCAT primary, secondary and tipping structure that exploits the excellent thermal and strength-to-weight properties of CFRP. The goal of this design is to demonstrate that a $3 \mathrm{D}$ distributed interface between 
the CFRP truss supporting the primary segments and the steel axle structure can be made very stiff without compromising the thermal performance. The truss structure is very deep and encloses the large $3 \mathrm{~m}$ diameter optical beam and instrument volume along the elevation axis.


Figure 2. Top, front, bottom and side views of the $25 \mathrm{~m}$ diameter CCAT tipping structure model. The model includes the primary and secondary mirrors and their support structure as well as the steel axle structure. The CFRP struts and primay segments are shown in gray. The steel tipping structure is shown in red (or dark gray). The optical beam and instrument volume are shown in tranlucent blue. The elevation drive bull gear is the seen in the side view. 
One of the challenges for this design was producing a sufficiently stiff secondary support structure while keeping the total optical blockage to less than $3 \%$. The result is a tripod structure with each leg of the tripod itself supported on a multi-legged structure attached to the primary truss. The near-in tripod legs are kept above the converging ray cone and hence only contribute to the incident plane wave blockage. The secondary support is fabricated from thin wall rectangular CFRP tubes with CTE designed to match that of the primary truss.

The steel tipping structure uses cylindrical beams and extends throughout a volume that is roughly $10 \mathrm{~m}$ on a side. The interface between the CFRP truss and steel structure is also distributed throughout this same volume and utilizes the thermal homology principles described in the previous section to minimize the thermal distortions. A close-up of this interface region is shown in figure 3 . There are 34 CFRP connecting struts that support loads in all directions and provide a very stiff transition between the CFRP truss and the steel axle structure. As described in the previous section, the choice for the center of expansion calculations is arbitrary but it is obviously convenient to pick the symmetry point at the intersection of the elevation and optical axis. Figure 3 illustrates how the CFRP connecting struts are perpendicular to the line from their attachment point on the steel structure to the chosen center of expansion.

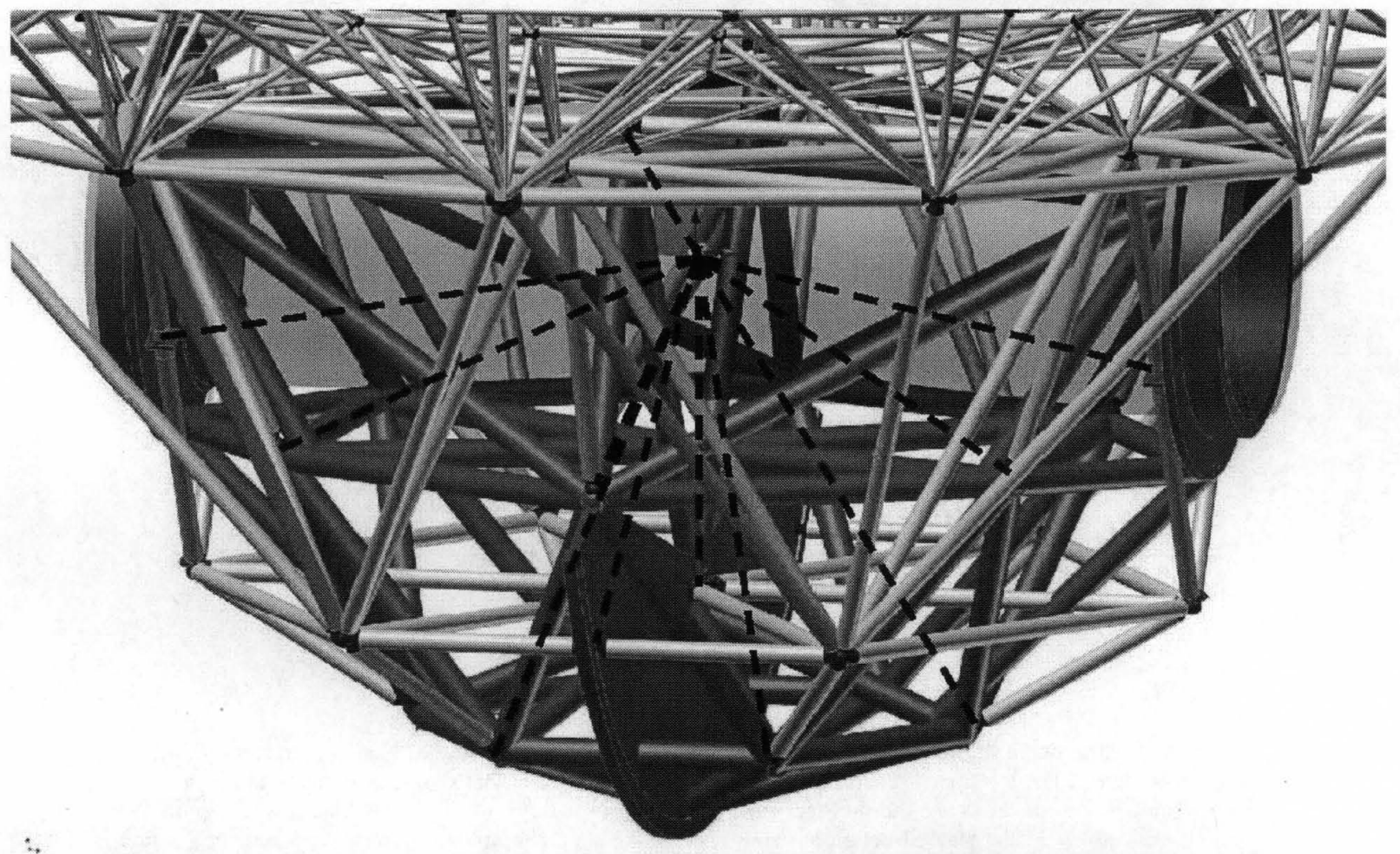

Figure 3. Close-up view of the interface between the CFRP structure and the steel axle structure. The CFRP struts are shown in gray. The steel tipping structure is shown in red (or dark gray). The CFRP struts that connect to the steel structure are perpendicular to the line from their attachment point to the chosen center of expansion as the intersection of the optical and elevation axes. These construction lines are drawn as dashed lines.

The requirement that the attachment struts be perpendicular to the line connecting the attachment point to the reference point does not fully constrain the strut orientation. Another constraint can be applied to accommodate different distortions of the steel structure. An example of this is used for the attachment to the elevation bearings as shown in figure 3. For these struts a second reference point is chosen at the center of the elevation bearing. The struts connecting to an elevation bearing are constrained to being perpendicular to both the line from the attachment point to the center of that elevation bearing as well as to the line from the attachment point to the intersection of axes. 
An alternate design for the steel tipping structure and interface to the CFRP truss is shown in figure 4 . The primary, secondary and all of the truss structure above the elevation axis are identical to the design and in figure 2 and are not drawn in figure 4. This design uses an augmented version of the ring of blades concept. The base of the CFRP truss has six nodes in a hexagon pattern which are connected to the steel tipping structure using steel blades. The stiffness of this connection is increased by adding CFRP struts from the six bottom nodes to the center of the hexagon where they connect to a central pillar on the steel structure. Thus instead of the usual $2 \mathrm{D}$ constraints provided by the blades at the corners of the hexagon there is a 3D constraint at these points. This design preserves the depth and stiffness of the CFRP truss using a simpler interface without jeopardizing the thermal performance. In addition the steel structure is compact, which makes thermal control of the steel structure more feasible. This will minimize thermal gradients in the steel structure and the distortions caused by these gradients.

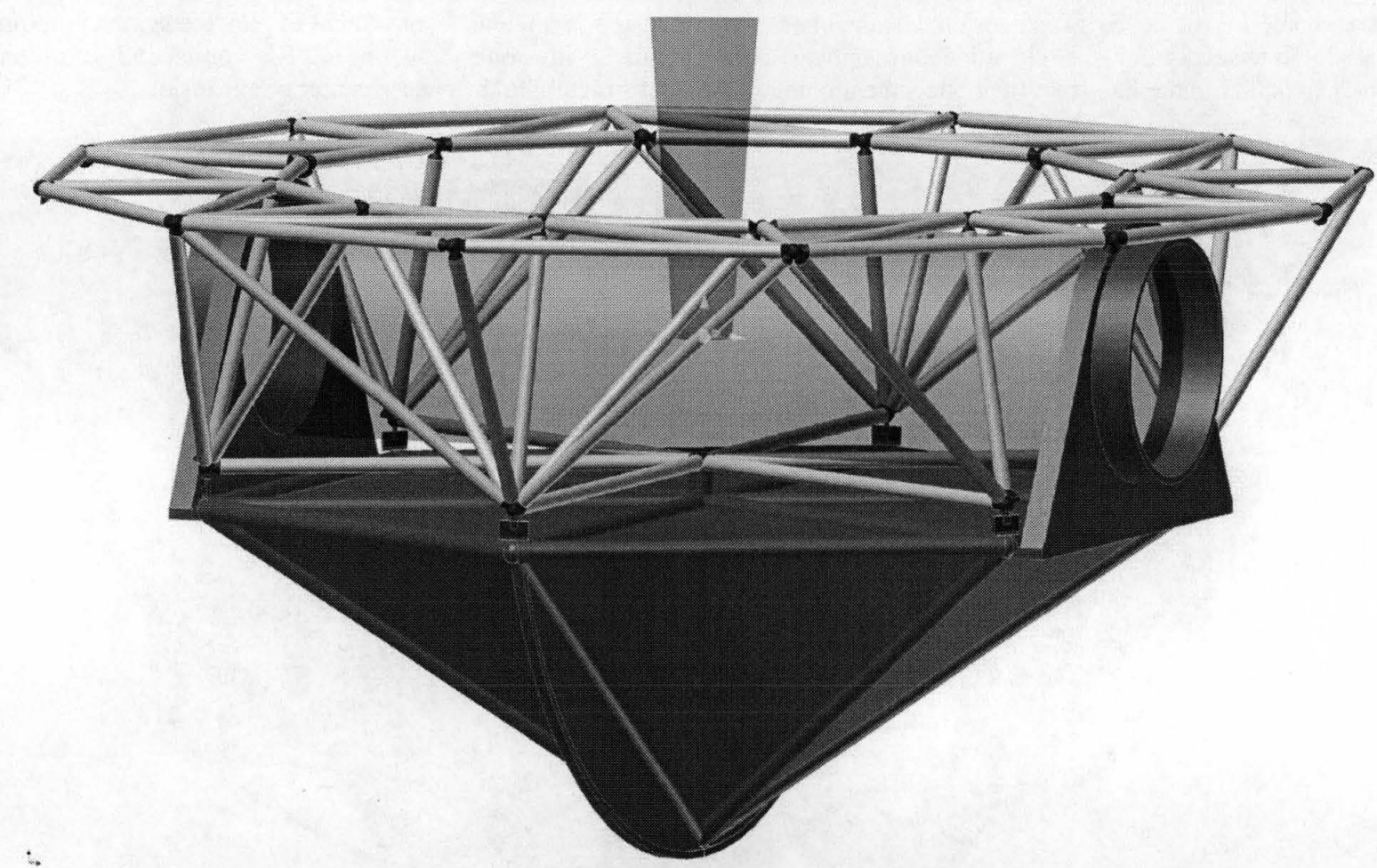

Figure 4. Central detail of a deep CFRP primary truss with an augmented ring of blades connection to the steel tipping structure. The top part of the primary CFRP truss is identical to that shown in figure 2 and is not shown here. The CFRP struts are gray and the steel structure is red (or dark gray). The steel blades which connect the bottom truss nodes to the steel structure are shown in dark blue. The optical beam and instrument volume is shown in translucent blue. A stiff steel central pillar in a plane below the elevation axis with CFRP struts connecting to the bottom nodes of the hexagon has been added to the standard ring of blades design to increase the stiffness without degrading the thermal performance. The expansion reference point is at the top of this central pillar.

\section{STRUT AND NODE DESIGN}

The design and fabrication of the CFRP struts and connecting nodes are critical to achieving the required performance. It is not sufficient to simply have CFRP tubes with low CTE. Most reliable and affordable connection methods utilize metallic end fittings and nodes. The mass and CTE of these metallic components can easily dominate both the thermal and gravity distortions of the structure. Achieving a high uniformity for the net effective CTE for all of the struts, including the end fittings and nodes can be more important than the average CTE for the structure, i.e. you don't want short struts to have a larger effective CTE than struts that are a few times longer. Passively achieving the CCAT 
specifications for performance for uniform temperature changes as large as $20 \mathrm{C}$ requires an effective CTE of $\sim 0.2$ $\mathrm{ppm} / \mathrm{C}$ with a uniformity of better than $0.1 \mathrm{ppm} / \mathrm{C}$.

CFRP tubes can be fabricated with different CTEs by varying the layup orientation and epoxy content of the tubes. But this is impractical for tailoring the CTE for the many dozens of different strut lengths and cross-sections. Invar alloys can have low CTEs in the range of $\sim 1 \mathrm{ppm} / \mathrm{C}$, much better than standard construction steels but still much worse than the $\sim 0.1 \mathrm{ppm} / \mathrm{C}$ for high quality CFRP tubes. So even using Invar for all of the metallic components is not sufficient to achieve a uniform effective CTE for all of the struts. In addition Invar is expensive and not readily available in the large variety of shapes and sizes that are needed.

Figure 5 shows the design of a strut with steel fittings that can be tailored to yield a uniform effective CTE for different length struts. It consists of an Invar node, stainless steel conical nose end pieces that are glued to a CFRP cone, and stainless steel coupling rings between the CFRP cone and the CFRP tube. The conical nose and rings are fabricated as separate inner and outer parts for ease of assembly. This construction minimizes the weight and effective expansion length for the metallic components. The net CTE for the strut is adjusted by tailoring the coupling rings to vary the distance from the large end of the CFRP cone to the CFRP tube end. Longer struts will have a larger gap between the two CFRP components effectively increasing the CTE for the strut to match the shortest struts which will have no gap. The same principle can be implemented or fine tuned by using different thicknesses of steel spacers between the conical nose fitting and the node ball. The $30 \mathrm{deg}$ conical taper of the end fitting allows a narrow opening angle between struts connecting to the same node which reduces the required size for the node ball. This is important for minimizing the mass and CTE.

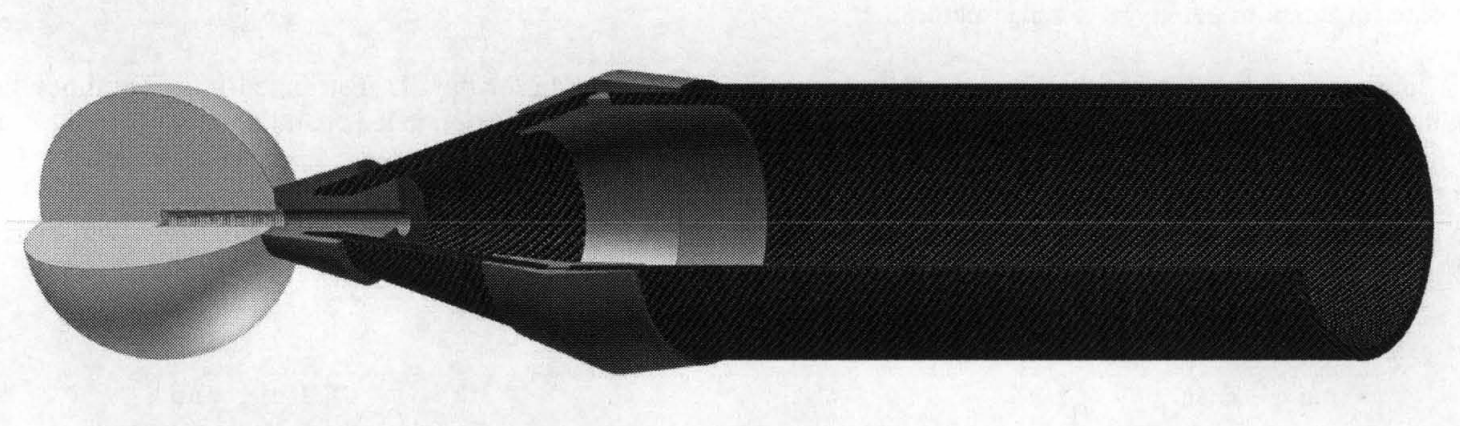

Figure 5. Node and strut with a CFRP conical end fitting. The black hatching is CFRP whereas the rest of the structure is metallic. The node ball is Invar and the coupling rings and conical end pieces are stainless steel. The conical steel end is two pieces that nest together for assembly as is the coupling ring. The effective CTE is adjusted by machining the coupling ring pieces to leave different gap lengths between the large end of the CFRP cone and the CFRP tube for the different length struts. The interior socket head bolt which fastens the strut to the node is not shown. This bolt is tightened using a ball-end wrench inserted through a slot in the CFRP tube.

\section{ANALYSIS}

The CCAT model described in section 3 has been analyzed as a truss structure using the Algor ${ }^{\mathrm{TM}}$ analysis program. The FEA includes most of the CFRP struts, secondary support, and the steel elevation structure. The details of the top layer of CFRP struts that interface to the actuators are not modeled but their mass is combined with the primary segment and actuator masses. The combination of these components is treated as lumped masses at the actuator nodes with a total effective areal density of $34 \mathrm{~kg} / \mathrm{m}^{2}$. The secondary is modeled as a $600 \mathrm{~kg}$ lumped mass at the prime focus position. Constraints appropriate for the elevation bearings and elevation drive system are applied to the nodes that connect to the elevation bearings and to a single node at the rim of the large elevation drive wheel.

Four classes of CFRP-Invar struts are used with cross-sectional areas of $0.01,0.005,0.0025$ and $0.001 \mathrm{~m}^{2}$. Each strut (CFRP tube with end fittings and nodes) was modeled as a single truss element with effective modulus of $2 \times 10^{11} \mathrm{~Pa}$, effective density of $3000 \mathrm{~kg} / \mathrm{m}^{3}$ and effective CTE of $0.2 \mathrm{ppm} / \mathrm{C}$. The parts counts and masses for the two designs 
described in section 3 are given in table 1. The steel tipping structure for the ring and pillar design is all below the elevation axis and beam cross-sections are adjusted to balance the telescope without requiring a separate counter weight.

Table 1. Tipping structure masses

\begin{tabular}{|l|l|r|r|r|r|}
\hline & & \multicolumn{2}{|c|}{ 3D distributed interface } & \multicolumn{2}{|c|}{ Ring and pillar interface } \\
\hline Item & Material & Quantity & Mass [kg] & Quantity & Mass [kg] \\
\hline Segments & Al and CFRP & 162 & 8,347 & 162 & 8,347 \\
\hline Actuators & Commercial component & 486 & 3,402 & 486 & 3,402 \\
\hline Strut ends and nodes & Invar and steel & 5,006 & 18,814 & 4,966 & 17,866 \\
\hline Strut tubes & CFRP & 2,469 & 17,314 & 2,483 & 16,434 \\
\hline Steel elevation structure & Steel & 179 & 31,511 & 41 & 38,865 \\
\hline Secondary & Al and CFRP & 1 & 600 & 1 & 600 \\
\hline Elevation drive wheel & Steel & 1 & 3,000 & 1 & 3,000 \\
\hline Counter weight & Steel & 1 & 13,000 & 1 & 0 \\
\hline Total tipping mass & & $\mathbf{9 5 , 9 8 8}$ & & $\mathbf{8 5 , 5 1 5}$ \\
\hline
\end{tabular}

The primary truss and axle models used in the FEA were transcribed from design models developed with the Autodesk Inventor $^{\mathrm{TM}}$ program. A few different sets of truss member cross-sections were investigated to arrive at a design with good performance. The secondary support design required several iterations of the geometry and cross-sections to achieve good performance. The following results should be conservative and further optimization should be able to compensate for the non-ideality of a real structure.

Table 2 summarizes the FEA results for the two models. The lowest mode for the 3D distributed interface model is shown in figure 6 and corresponds to tipping about the Y-axis. Note that the elevation axle is parallel to the X-axis. The lowest mode for the ring and pillar interface model has the same shape. The high resonant frequencies bode well for meeting the tracking and pointing specifications. The gravity distortions greatly exceed the required surface error but will be corrected by using lookup tables. This still requires that the gravity distortions be known and stable with better than $1 \%$ accuracy.

Table 2. FEA results.

\begin{tabular}{|l|r|r|}
\hline Analysis case & $\begin{array}{l}\text { 3D } \\
\text { distributed } \\
\text { interface }\end{array}$ & $\begin{array}{l}\text { Ring and } \\
\text { pillar } \\
\text { interface }\end{array}$ \\
\hline Lowest resonance & $\begin{array}{r}10.1[\mathrm{~Hz}] \\
\text { RMS } \\
\text { distortion }\end{array}$ & $\begin{array}{r}\text { RMS } \\
\text { distortion }\end{array}$ \\
\hline Z-gravity & $128[\mu \mathrm{m}]$ & $112[\mu \mathrm{m}]$ \\
\hline$Y$-gravity & $148[\mu \mathrm{m}]$ & $218[\mu \mathrm{m}]$ \\
\hline$\Delta \mathrm{T}=10 \mathrm{C}$ temperature change & $2.1[\mu \mathrm{m}]$ & $0.1[\mu \mathrm{m}]$ \\
\hline 0.1 ppm/C CTE random variation \& 10C temperature change & $5.9[\mu \mathrm{m}]$ & $5.9[\mu \mathrm{m}]$ \\
\hline$\Delta \mathrm{T}=1 \mathrm{C}$ front-to-back & $3.1[\mu \mathrm{m}]$ & $3.1[\mu \mathrm{m}]$ \\
\hline$\Delta \mathrm{T}=1 \mathrm{C}$ front-to-back, refocused & $0.2[\mu \mathrm{m}]$ & $0.2[\mu \mathrm{m}]$ \\
\hline$\Delta \mathrm{T}=1 \mathrm{C}$ top-to-bottom & $1.5[\mu \mathrm{m}]$ & $1.5[\mu \mathrm{m}]$ \\
\hline$\Delta \mathrm{T}=1 \mathrm{C}$ top-to-bottom, repoint & $0.1[\mu \mathrm{m}]$ & $0.1[\mu \mathrm{m}]$ \\
\hline$\Delta \mathrm{T}=+-0.5 \mathrm{C}$ random in CFRP truss & $0.6[\mu \mathrm{m}]$ & $0.6[\mu \mathrm{m}]$ \\
\hline$\Delta \mathrm{T}=+-0.5 \mathrm{C}$ random in steel axle structure & $2.4[\mu \mathrm{m}]$ & $6.7[\mu \mathrm{m}]$ \\
\hline
\end{tabular}

The RMS distortion is calculated after correcting for focus and pointing

The use of a CFRP truss dramatically reduces the thermal distortions but these distortions are significant and extra steps are required to meet the demanding CCAT specifications. The effect of a uniform temperature change of $10 \mathrm{C}$ for the $3 \mathrm{D}$ distributed interface model is shown in figure 7 . The steel axle shows large distortion but results in only a 2.1 
micron RMS surface distortion. This is larger than expected from equ. 1 and is the result if imperfectly transcribing the interface attachment geometry from the Inventor ${ }^{\mathrm{TM}}$ model to the FEA model. The actual construction errors could be similar to these transcription errors and a coarse distortion vs. temperature lookup table may be required. The geometry for the ring and pillar interface is much simpler, making accurate modeling and construction easy, resulting in only a 0.1 micron RMS surface distortion for a $10 \mathrm{C}$ temperature change.

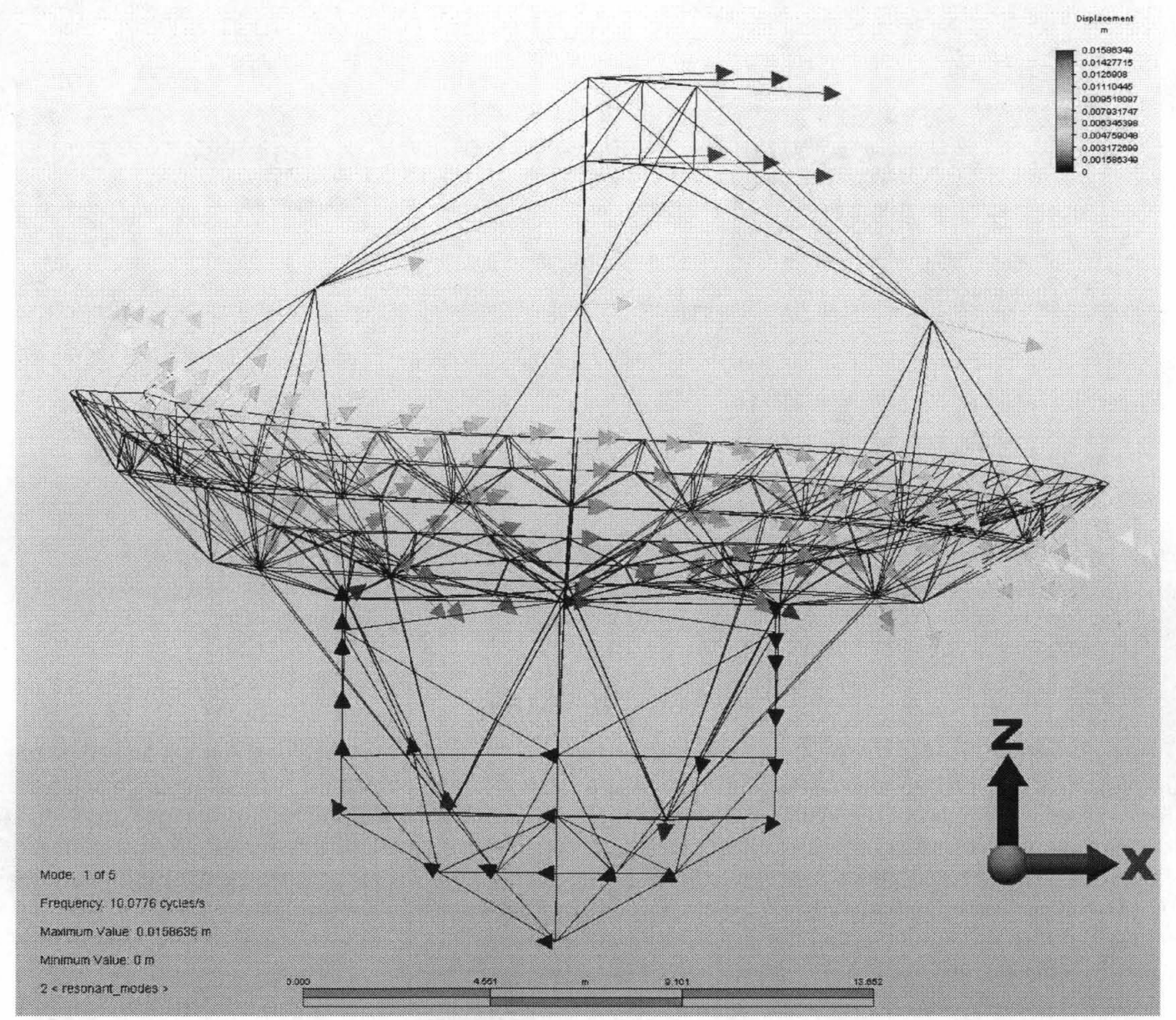

Figure 6. View from the front of the mode shape for the $10.1 \mathrm{~Hz}$ lowest resonant mode for the 3D distributed interface model.

As noted in the previous section the uniformity of the effective CTE is important and a large source of thermal distortion. Fortunately this error can be corrected using a coarse table of adjuster settings vs. ambient temperature. Although many of the other thermal distortions can also be corrected by characterizing the surface as a function of many temperature readings across the structure, this would require extensive modeling and measurements of the surface in operation.

Random temperature variations across the steel axle structure will need to be kept less than $\sim 1 / 2 \mathrm{C}$ for the 3D distributed interface model. The ring and pillar model has few components than the steel structure which actually increases the effect of temperature variations and requires correspondingly better temperature uniformity. Fortunately the simplicity and compactness of this steel structure makes temperature regulation relatively easy. 




Figure 7. View from the front of the distortions for a uniform temperature change of $10 \mathrm{C}$. The RMS surface distortion for this load case is 1 micron. The arrows indicate the direction and magnitude of the distortions. The distortions range from 0 to $1.6 \mathrm{~mm}$.

\section{SUMMAY}

A conceptual design for the CCAT telescope has been developed that uses the excellent thermal and high strength to weight properties of CFRP to meet the stringent surface accuracy for the primary. This design demonstrates that it is feasible to use the principles of thermal homology to design very stiff and robust connections between large structures of dissimilar materials that preserve the excellent thermal performance of an all CFRP structure. Also a design for the CFRP struts, end fitting and nodes is presented that addresses the problem of fabricating many different strut crosssections and lengths with the same effective low CTE. FEA of the structure indicates that it should meet the stringent 6 micron RMS primary distortion requirement under the expected operating conditions using lookup tables to correct the gravity deflections and distortions resulting from large temperature changes.

\section{ACKNOWLEDGEMENTS}

This work was supported by the John B. and Nelly Kilroy Foundation.

\section{REFERENCES}

[1] Sebring, T.A., The Cornell Caltech Atacama Telescope: progress and plans 2010. Proc. SPIE. 7733 (2010).

[2] Padin, S., et al., CCAT Optics. Proc. SPIE. 7733 (2010).

[3] Woody, D.P., et al., Panel options for large precision radio telescopes. Proc. SPIE. 7018: p. OT1-OT11 (2008).

[4] Redding, D.C., et al., Wavefront controls for a large submillimeter-wave observatory Proc. SPIE. 7733 (2010).

[5] Lou, J.Z., et al., Modeling a large submillimeter-wave observatory Proc. SPIE. 7733 (2010).

[6] Greve, A., M. Dan, and J. Penalver, Thermal behavior of millimeter wavelength radio telescopes. IEEE Transactions on Antennas and Propagation. 40(11): p. 1375-1388 (1992).

[7] Baars, J., W. M., ed. The Paraboloidal Reflector Antenna in Radio Astronomy and Communication. Astrophysics and Space Science Library, ed. W.B. Burton. 2007, Springer: New York, NY. 